OPEN ACCESS

Edited by:

Mo Ziaei,

The University of Auckland,

New Zealand

Reviewed by:

Isabella Cheung,

The University of Auckland,

New Zealand

Jianqiao Li,

Shandong University, China

Honghua Yu,

Guangdong Provincial People's

Hospital, China

*Correspondence:

Zhenzhen Liu

liuzhenzhen@gzzoc.com

Lixia Luo

Iuolixia@gzzoc.com

tThese authors have contributed equally to this work and share first

authorship

Specialty section:

This article was submitted to

Ophthalmology,

a section of the journal

Frontiers in Medicine

Received: 15 September 2021

Accepted: 10 January 2022

Published: 14 February 2022

Citation:

Ruan X, Liang C, Xia Z, Tan X, Jin G, Jin L, Liu Z and Luo L (2022) In-vivo

Lens Biometry Using the Novel

Ultrasound Biomicroscopy.

Front. Med. 9:777645.

doi: $10.3389 /$ fmed.2022.777645

\section{In-vivo Lens Biometry Using the Novel Ultrasound Biomicroscopy}

\author{
Xiaoting Ruan ${ }^{1+}$, Chen Liang ${ }^{1 \dagger}$, Zhaoxia Xia ${ }^{2 \dagger}$, Xuhua Tan ${ }^{1}$, Guangming Jin ${ }^{1}$, Ling Jin ${ }^{1}$, \\ Zhenzhen Liu $^{1 *}$ and Lixia Luo ${ }^{1 *}$ \\ ${ }^{1}$ State Key Laboratory of Ophthalmology, Zhongshan Ophthalmic Center, Sun Yat-sen University, Guangdong Provincial Key \\ Laboratory of Ophthalmology and Visual Science, Guangdong Provincial Clinical Research Center for Ocular Diseases, \\ Guangzhou, China, ${ }^{2}$ Department of Ophthalmology, The Sixth Affiliated Hospital, Sun Yat-sen University, Guangzhou, China
}

Background and Aim: To assess the reproducibility of the novel ultrasound biomicroscopy, Insight 100 and its agreement with a swept-source optical coherence tomography, CASIA2.

Methods: A total of 96 volunteers (96 eyes) were enrolled. The radius of anterior lens curvature (RAL), the radius of posterior lens curvature (RPL), lens thickness ( $L T)$, and lens diameter (LD) were measured with Insight 100 and CASIA2. A semiautomated software was used to adjust the measurement of $L T\left(L T_{S}\right)$ and $L D\left(L D_{S}\right)$ by Insight 100. Intraobserver and interobserver reproducibility of Insight 100 measurements, and the agreement of results from Insight 100 and CASIA2 were assessed with 95\% limit of agreement (LOA), intraclass correlation coefficient (ICC), Pearson correlation, and linear regression.

Results: For Insight 100 measurements, the intraobserver ICCs of RAL, RPL, LTS, and LDS measurement were 0.996, 0.973, 0.936, and 0.889, and the interobserver ICCs were 0.987, 0.890, 0.974, and 0.816, respectively. There was an excellent correlation in LT measurements $(R=0.961, P<0.001)$ but poor agreements in other parameters between the two devices. The LD measurements tended to be larger $(95 \%$ Cl: 0.768-0.928) in CASIA2 when compared with Insight 100.

Conclusion: Insight 100 could obtain highly repeatable lens biometry in vivo. With better signal penetration, it shows promising potential in future clinical applications.

Keywords: ultrasound biomicroscopy (UBM), anterior segment, ОCT, lens biometry, lens curvature, lens thickness, lens diameter

\section{INTRODUCTION}

The measurement of lens parameters is of great importance in both research of lens function, and calculation of the intraocular lens (IOL) power (1-5). Currently, the commercially available devices providing in vivo lens parameters measurement include the Scheimpflug photography, anterior segment optical coherence tomography (AS-OCT) and ultrasound biomicroscopy (UBM) (6-10). Among them, the latter two could provide a full set of in-vivo lens biometry.

The new generation swept-source optical coherence tomography (SS-OCT), CASIA2, can provide the automatic noncontact measurement of lens parameters using a built-in program. But since the light source of CASIA2 cannot penetrate the pigmented iris, the detection area is limited $(7,8)$. The conventional UBM with deeper signal penetration could image the structures behind 
the iris where the light cannot reach. However, this contact measurement is time-consuming and less convenient (9). The newly developed very high frequency (VHF) UBM, Insight 100 using a disposable eyepiece is less invasive than the conventional UBM. It also has the advantage over the optical device of better signal penetration. With a better view of the peripheral lens behind the iris, the Insight 100 may show more potential in ensuring more accurate measurements of the lens parameters especially for patients with relative contraindications of contact examination or being contraindicated of pupil dilation.

To provide more evidence if this novel UBM device could be a potential tool for in vivo lens biometry, this study investigated the intraobserver and interobserver reproducibility of the Insight 100 , and its agreement with the commercially available CASIA2.

\section{MATERIALS AND METHODS}

\section{Participants}

This study was conducted at the Zhongshan Ophthalmic Center, Sun Yat-sen University, Guangzhou, China. Volunteers were consecutively recruited from the Outpatient Department from January to June 2020. Subjects with any evidence of the following conditions were excluded: 1) ocular disease besides senile cataract and refractive error; 2) history of intraocular surgery; and 3) inability to cooperate with the test, or poor fixation resulting in low image quality. The study was approved by the ethics committee of Zhongshan Ophthalmic Center and was performed following the tenets of the Declaration of Helsinki. Written informed consents were obtained from all participants.

\section{Anterior Segment Scanning}

Anterior segment scanning was performed with SS-OCT, CASIA2 (Tomey Corporation, Nagoya, Japan) and the UBM, Insight 100 (ArcScan Incorporation, Morrison, Colorado, USA). These two devices were operated independently by two experienced operators (XT-R and C-L) in random sequence. The operators were masked to results of one another. Neither mydriatic nor miotic drops were applied before the test, to avoid any accommodation stimulus. Scanning by the two devices was performed in the same room and under the same lighting conditions. Fixation target is consistent during measurements in CASIA2 and Insight 100.

\section{CAISA2 Scanning}

The CASIA2 uses a swept-source laser with a wavelength of $1,310 \mathrm{~nm}$ at a velocity of 50,000 A-scan/s. The axial and transverse resolutions are $10 \mu \mathrm{m}$ or less and $30 \mu \mathrm{m}$ or less, respectively. The participants were seated and asked to fixate on the external lights during the examination. Lens biometric parameters including radius of anterior lens surface curvature (RAL), the radius of posterior lens surface curvature (RPL), lens thickness (LT), and lens diameter (LD) were automatically measured by the builtin software (Version 3G.1). The CASIA2 measurements of lens biometry were aligned along the visual axis and performed using the 16-scan "lens biometry" mode. Then the measurement results were automatically generated by the "Lens Analysis" module in "Lens Biometry" mode. After the measurement, the operator can check the clarity and fixation of all images in the preview, after which the qualified measurements were selected for analysis. The 2D analysis results of 0-180 degree in "Len Biometry" mode was used for the comparison with UBM.

\section{Insight 100 Scanning}

The Insight 100 examination for lens biometry was performed using the "capsule" mode with a broadband 20-60 MHz VHF ultrasound transducer. This system has a tissue penetration depth of $15 \mathrm{~mm}$. The entire anterior segment is presented in a single image with an axial resolution of $35 \mu \mathrm{m}$ and lateral resolution of $65 \mu \mathrm{m}$. The scan is angular (2 scans/s); the image area is adjustable by the user up to 70 degrees and $22 \mathrm{~mm}$. The ultrasound velocity in this study was set at 1,640 m/s for the lens capsule. The capsular bag was examined on the axial horizontal section (transverse diameter passing through the corneal apex from 9 to 3 o'clock). During UBM scanning, the participants were seated with the chin and forehead placed into a headrest. A soft rimmed eye-cup was placed in the eye to be examined, with a soft membrane separating the eye from the transducer and scanning chamber, which was filled with distilled water. Participants fixated with the fellow eye on a narrow fixation target to ensure that the corneal vertex was coaxial with the infrared camera and the scanning rotation center. The examiner clicked on the video feed of the eye to adjust the system to be centered on the corneal reflex. The automated centration algorithm used information from horizontal and vertical scans to find the corneal vertex. The measurement achieved quality control by built-in software for statistical analysis.

The scenario of the Insight 100 examination is illustrated in Supplementary Figure S1. The acquisition with the qualified fixation was selected for further analysis of crystalline lens biometry.

\section{Manual Measurement Using Insight 100 Images}

Two ophthalmologists (XT-R and C-L) independently measured the RAL, RPL, LT, and LD in the images by using the built-in manual caliper tool of UBM. The built-in manual caliper tool can generate a fitting arc through three points. The RAL was determined as follows: the first two points were defined as where the anterior lens capsule intersects with the iris, and the third point was the apex of the anterior lens capsule. The RPL is determined similarly to the above three points. The fitting curve could be adjusted via moving these three points for the best fit. The LT was defined as the distance between apexes of the anterior and posterior lens surfaces. The LD was defined as the distance between the intersections of the anterior and posterior lens surface fitting curves. Each selected scan was measured by one ophthalmologist repeating three times.

\section{Semiautomated Measurement Using Insight 100 Images}

After input of the contour line and curvature of the anterior and posterior lens surface, the customized developed software can automatically process further segmentation and fitting based on the input data. After adjustment, more accurate contour lines of 
A

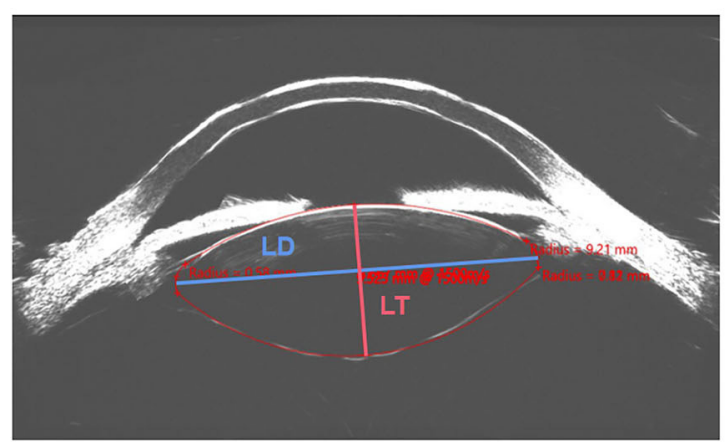

B

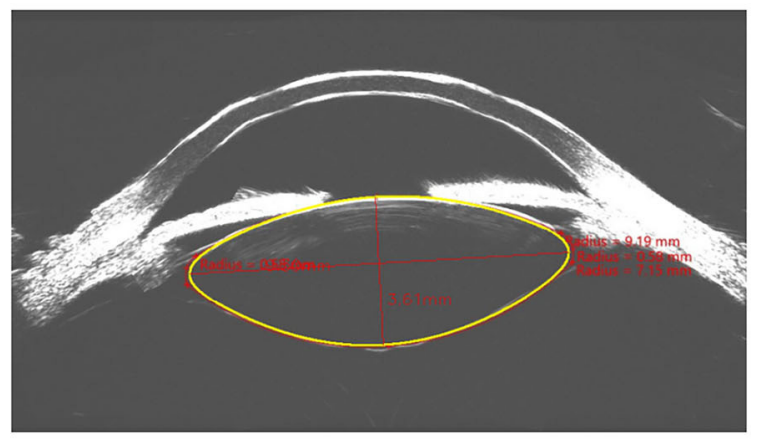

FIGURE 1 | Manual measurement (A) and semiautomated measurement (B) of the lens diameter (LD) and lens thickness (LT) in the same image obtained by Insight 100.

TABLE 1 | Interobserver reproducibility of lens biometry measurements with Insight 100.

\begin{tabular}{|c|c|c|c|c|c|c|c|}
\hline & & \multirow[t]{2}{*}{ Mean $\left(\mathrm{mm}^{3}\right)$} & \multirow[t]{2}{*}{$\mathrm{SD}\left(\mathrm{mm}^{3}\right)$} & \multirow[t]{2}{*}{ Mean difference $(95 \% \mathrm{Cl})$} & \multirow[t]{2}{*}{$P$ value } & \multicolumn{2}{|c|}{ Interobserver reproducibility } \\
\hline & & & & & & 95\%LoA & ICC \\
\hline \multirow[t]{2}{*}{ RAL } & Observer 1 & 10.308 & 1.530 & $-0.079(-0.128$ to -0.030$)$ & $0.002^{*}$ & -0.392 to 0.549 & 0.987 \\
\hline & Observer 2 & 10.229 & 1.562 & & & & \\
\hline \multirow[t]{2}{*}{ RPL } & Observer 1 & 6.388 & 0.752 & $0.142(0.077$ to 0.207$)$ & $<0.001^{*}$ & -0.770 to 0.486 & 0.890 \\
\hline & Observer 2 & 6.530 & 0.724 & & & & \\
\hline \multirow[t]{2}{*}{$\mathrm{LT}_{\mathrm{S}}$} & Observer 1 & 3.564 & 0.345 & $0.022(0.006$ to 0.037$)$ & $0.006^{\star}$ & -0.170 to 0.127 & 0.974 \\
\hline & Observer 2 & 3.586 & 0.342 & & & & \\
\hline \multirow[t]{2}{*}{$\mathrm{LD}_{\mathrm{S}}$} & Observer 1 & 9.032 & 0.387 & $0.115(0.074$ to 0.157$)$ & $<0.001^{*}$ & -0.516 to 0.286 & 0.816 \\
\hline & Observer 2 & 9.148 & 0.368 & & & & \\
\hline
\end{tabular}

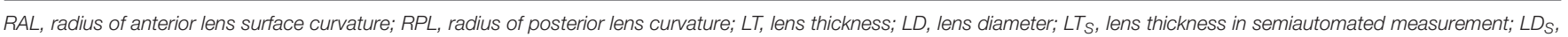
lens diameter in semiautomated measurement; LOA, the limit of agreement; ICC, intraclass correlation coefficient.

Bold*: Statistically significant difference $(P<0.05)$.

the anterior, posterior, left, and right surface of the lens were obtained generating semiautomated measurements of the lens thickness and lens diameter $\left(\mathrm{LD}_{\mathrm{S}}\right.$ and $\left.\mathrm{LT}_{\mathrm{S}}\right)$. Figure 1 shows the manual measurement and semiautomated measurement of the $\mathrm{LD}_{\mathrm{S}}$ and $\mathrm{LT}_{\mathrm{S}}$ in the Insight 100 scan of the same eye.

\section{Statistical Analysis}

The sample size was calculated based on the interobserver agreement of Insight 100, which was assessed using intraclass correlation (ICC). A sample of 93 subjects who were each measured 2 times was necessary to estimate the ICC of 0.8 and a two-sided 95\% CI with a width of 0.100 , using a twoway random-effects ANOVA model. The PASS 16.0 (NCSS, LLC, Kaysville, Utah, USA) was used to calculate the sample size.

With the binocular parameters comparable in subjects, the right eyes were chosen for analysis. All continuous variables were expressed as mean \pm SD. The ICCs and the 95\% limit of agreement (LoA) were used to assess intraobserver and interobserver reproducibility of the manual measurements of RAL, RPL, and semiautomated measurement of $\mathrm{LT}_{\mathrm{S}}, \mathrm{LD}_{\mathrm{S}}$ using Insight 100 scans. The ICC estimates and their 95\% CIs were calculated based on an absolute-agreement, two-way randomeffects model. For each eye, the comparison was made between the average lens parameters of three repeated Insight 100 scans and the $2 \mathrm{D}$ result in a horizontal position from CASIA2. The 95\% LoA, the ICC (two-way random model), Pearson correlation analysis, and Bland-Altman plot were used for comparing the agreement between measurements from the Insight 100 and the CASIA2. The linear regression was used to evaluate the conversion of lens biometry between the Insight 100 and the CASIA2. All statistical analyses were performed using SPSS statistical software (SPSS Statistics version 22.0; IBM Corp., Armonk, NY, USA). Statistical significance was defined as $P<0.05$.

\section{RESULTS}

In this study, 96 eyes from 96 volunteers (40 male and 56 female) were included. The mean age of all participants was $34.42 \pm 11.19$ years old, ranging from 23 to 74 years old. Baseline characteristics of the participants and the pipeline of the study were demonstrated in Supplementary Figure S2 and Supplementary Table S1. All participants had completed both CASIA2 and UBM tests.

Two examiners performed measurements for all participants using Insight 100 scans. There were excellent intraobserver 


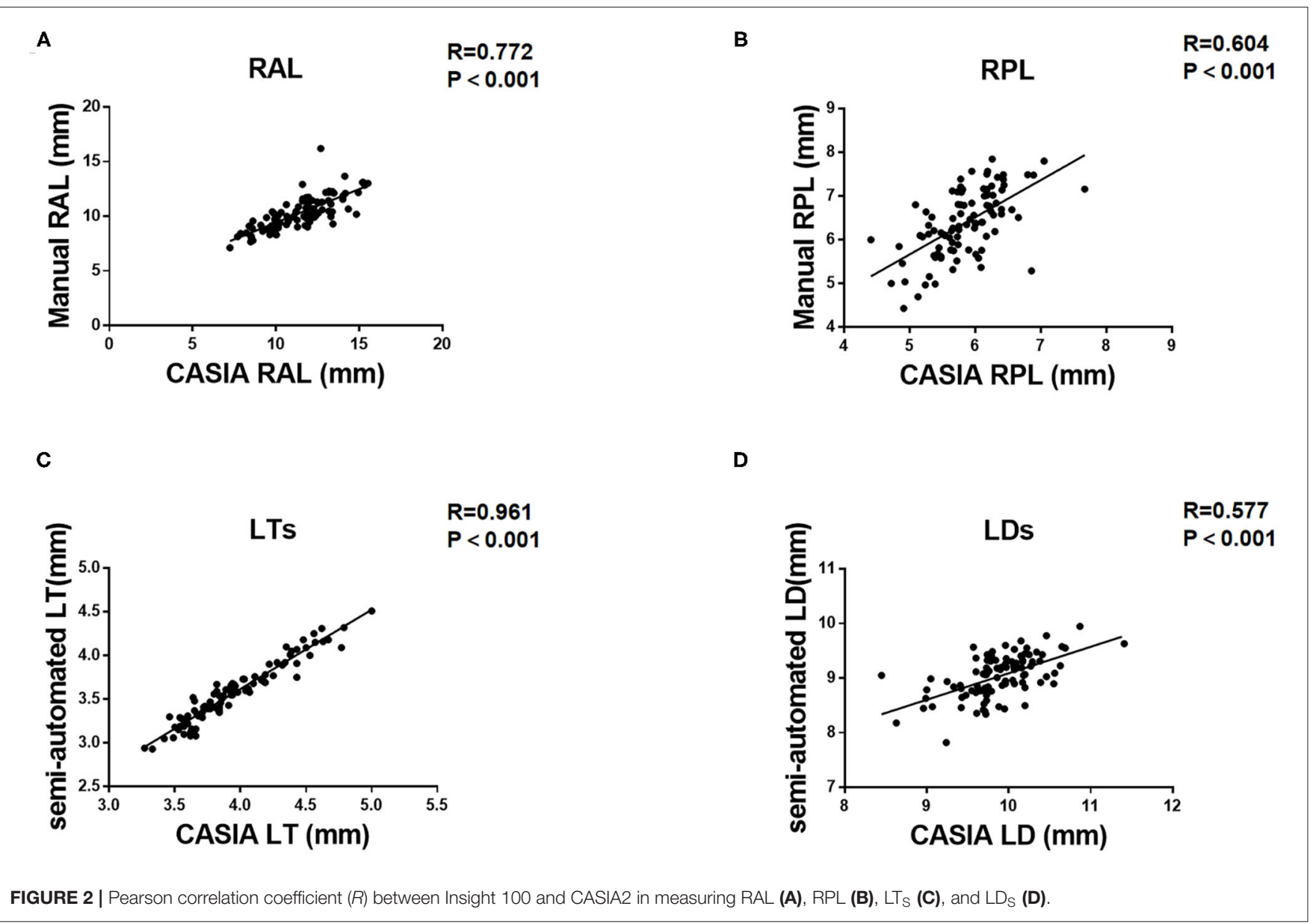

repeatabilities for manual measurements of RAL, RPL, and semiautomated measurements of $\mathrm{LT}_{\mathrm{S}}$ and $\mathrm{LD}_{\mathrm{S}}$ (ICC: 0.996, $0.973,0.936$, and 0.889 , respectively). And as shown in Table 1, the interobserver reproducibilities were excellent in measuring RAL and LT $_{S}$ (ICC: $0.987,0.974$, respectively) and good in measuring RPL and LDS (ICC: 0.890, 0.816, respectively).

As for the agreement of Insight 100 and CASIA2, the Pearson correlation coefficient $(R)$ in measuring RAL, RPL, LT, and LD was $0.772(P<0.001), 0.604(P<0.001), 0.961$ $(P<0.001)$, and $0.577(P<0.001)$, respectively (Figure 2$)$. According to the Bland-Altman analysis shown in Figure 3, the CASIA2 gave larger measures in lens thickness and lens diameter than that from the semiautomated measurements from Insight 100. And these differences tended to be consistent across the measurement ranges. The linear regression equations for measurement conversion were as follows ( $\mathrm{Y}$ represented measurement using the Insight 100, X represented measurement using the CASIA2):

$$
\begin{aligned}
\mathrm{RAL}: \mathrm{Y}_{\text {Insight100 }} & =0.6126^{*} \mathrm{X}_{\text {CASIA2 }}+3.300(\mathrm{P}<0.001) \\
\mathrm{RPL}: \mathrm{Y}_{\text {Insight100 }} & =0.8503^{*} \mathrm{X}_{\text {CASIA2 }}+1.415(\mathrm{P}<0.001) \\
\mathrm{LT}: \mathrm{Y}_{\text {Insight100 }} & =0.9076^{*} \mathrm{X}_{\text {CASIA2 }}-0.01326(\mathrm{P}<0.001) \\
\mathrm{LD}: \mathrm{Y}_{\text {Insight100 }} & =0.4845^{*} \mathrm{X}_{\text {CASIA2 }}+4.245(\mathrm{P}<0.001)
\end{aligned}
$$

Table 2 shows the comparison of lens biometry measured with the CASIA2 and the Insight 100. The 95\% LoA between the CASIA2 and the Insight 100 was -3.537 to $1.271 \mathrm{~mm}$ (RAL), -0.646 to $1.724 \mathrm{~mm}$ (RPL), $0.179-0.575 \mathrm{~mm}$ (LT), $0.072-$ $1.623 \mathrm{~mm}$ (LD); and the ICCs were $0.622,0.427,0.615$, and 0.191 for the above-mentioned parameters, respectively.

\section{DISCUSSION}

As a less invasive novel VHF UBM, Insight 100 showed excellent intraobserver and interobserver reproducibility in measuring RAL, RPL, LT, and LD, which has not been reported by previous studies. There was an excellent agreement between Insight 100 and CASIA2 in measuring lens thickness, with a relatively poor agreement in the measurement of RAL, RPL, and lens diameter. And measurements of lens thickness and diameters from Insight 100 tended to be smaller when compared with those from CASIA2.

By now, there is no golden standard of the in vivo lens biometry, that comparisons among different devices are still inconclusive if which one would be the most accurate. Several studies have been conducted to investigate the performance of different devices, which are summarized in Table 3 . In general, results from CASIA2 were proved to be reproducible, with better 
A

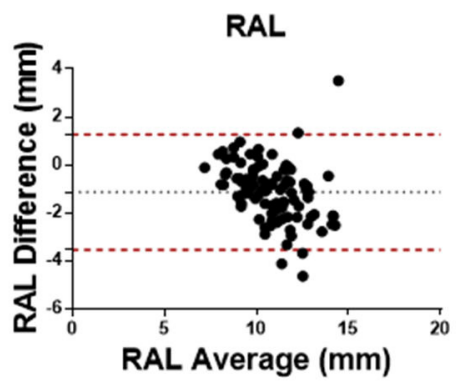

C

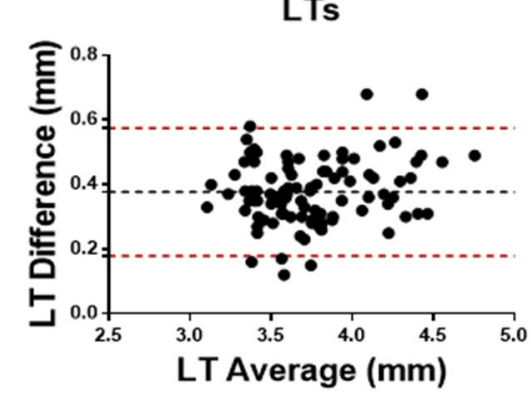

B

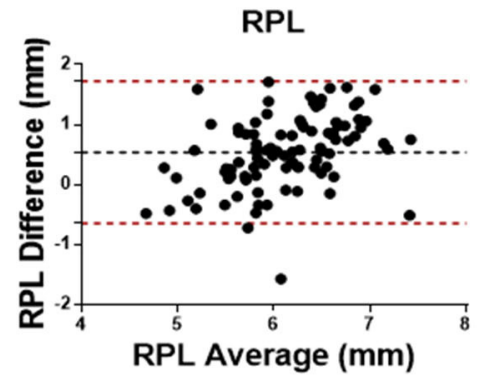

D

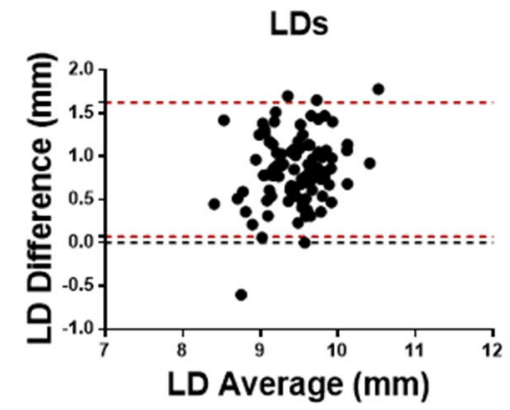

FIGURE 3 | Bland-Altman analysis between Insight 100 and CASIA2 in measuring RAL (A), RPL (B), LTS (C), and LDS (D).

TABLE 2 | Comparison of lens biometry measured with CASIA2 and Insight 100.

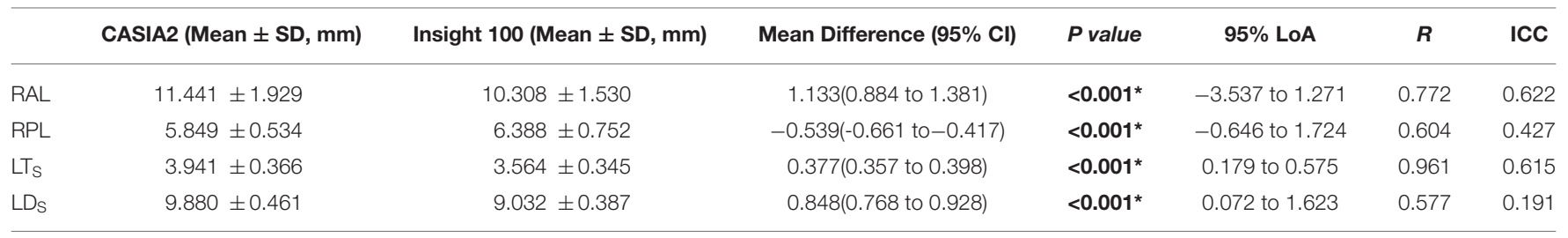

$R A L$, radius of anterior lens surface curvature; $R P L$, radius of posterior lens curvature; $L T_{S}$, lens thickness in semiautomated measurement; $L D_{S}$, lens diameter in semiautomated measurement; LOA, the limit of agreement; ICC, intraclass correlation coefficient; Bold": Statistically significant difference $(P<0.05)$.

performance in younger individuals. The RAL measurements could be interchangeable between CASIA2 and Scheimpflug imaging. While the measurement of the posterior lens was shown to be less stable, which could be affected by mild cataract. Also, the correlation was poor in measuring the posterior lens between CASIA2 and biometry with better penetration, for example, the ultrasound-based Insight 100.

Using different imaging principles, the measurements of Insight 100 and CASIA2 would be affected by different factors. The optical CASIA2 requires correction of optical distortion from the cornea and aqueous humor when measuring the anterior lens surface; and measurement of the posterior lens surface would be further affected by the heterogeneity of refractive indices of the lens (9). Insight 100 is an ultrasound device with better signal penetration. The difference in imaging principles and signal penetration may explain the poor agreement in anterior lens surface measurement, and the even poorer agreement in measuring posterior lens surface. On the other hand, while inconsistent reproducibilities in measuring posterior lens surface by CASIA2 have been reported, the Insight 100 showed excellent intraobserver repeatability and good interobserver reproducibility in measuring RPL according to the current study $(7,8)$.

There have been limited reports on the measurement of lens diameter, which is an important assessment for postoperative IOL stability $(3,11)$. CASIA2 using infrared light unable to penetrate the iris can only detect the lens limited to the pupil area. And the measurement of lens diameter in CASIA2 is based on a simulated image with sharp peripheral lens angles, which surely does not match with the real shape of the human lens. The Insight 100 can detect the area behind the iris, providing a better view of the peripheral lens. By further processing with the semiautomated software, the adjusted peripheral contour lines present a more accurate ellipsoid-shape lens rather than 


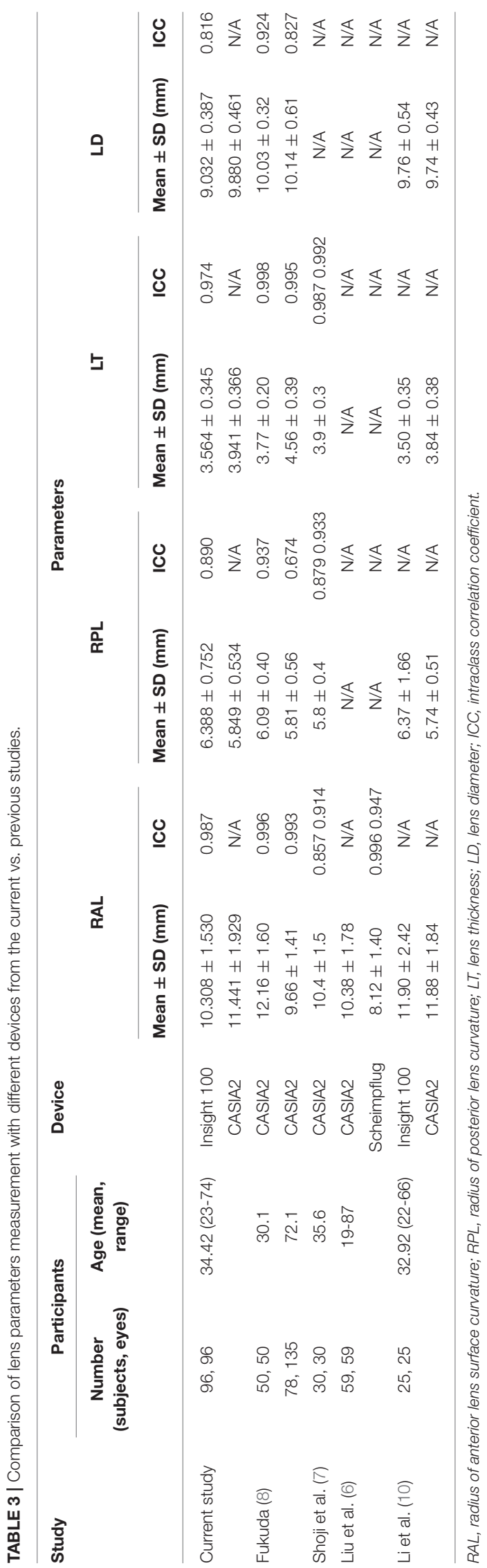

a spindle-shaped one in CASIA2. This would explain why the measurements of lens diameter in Insight 100 were smaller than those in CASIA2. On the other hand, the semiautomated software showing good reproducibility in measuring lens diameter may be promising in clinical assessment, though further clinical studies are still needed.

While measurement of the lens surface curvature and lens diameter would be affected by the lens periphery, the measurement of lens thickness could be achieved based on the central part of the lens even with limited visualization of the periphery. This could explain the good agreement of Insight 100 with CASIA2. Moreover, high reproducibilities of lens thickness measurement in different devices were also reported previously (7-9). The lens thickness is proved to be correlated with postoperative IOLs rotational stability (4). Therefore, Insight 100 also showed potential in future clinical assessment.

When compared with previous studies, the sample size of our study is relatively large (96 eyes from 96 subjects, larger than that in the study by Liu et al. (6), Shoji et al. (7), and Li et al. (10), but smaller than that in the study by Fukuda et al. (8), with a wide age range (23-74 years old). To the best of our knowledge, this study is the first study to report the reproducibility of the novel UBM, Insight 100 in measurements of lens biometry in vivo. Results suggested that Insight 100 could provide highly repeatable measurements of the lens parameters in vivo. Based on a simulated image that was closer to the real lens shape, stable measurement of lens diameter could be obtained after further processing with semiautomated software. Agreement between Insight 100 and CASIA2 was good in measuring lens thickness, but poor in anterior and posterior lens curvature and lens diameter measurement. With better visualization of the peripheral lens, Insight 100 would be promising as an in vivo biometry in real clinical practice.

The limitation of the Insight 100 should be considered for further clinical application of the device. First, Insight 100 requires a manual caliper while CAISA2 can calculate the result automatically with a built-in program. However, the intraobserver and interobserver reproducibility of Insight 100 were high among all lens parameters, indicating Insight 100 could provide repeatable results. Second, a qualified evaluation of the accommodation during the Insight 100 test was unavailable. In this study, only healthy individuals were included. With higher signal penetration depth superior to the optical device, the UBM would be more preferable in patients with refractive media opacity, for example, corneal scar. Further study including subjects with other ocular diseases (e.g., corneal opacity, cataract of various stages) and subgroup analysis would be investigated to better evaluate the effectiveness and clinical potential of Insight 100.

\section{CONCLUSION}

The novel VHF UBM, Insight 100 could provide repeatable full-set in-vivo lens parameter measurements. It showed good agreement with the commercially available SS-OCT in measuring 
lens thickness, but poor agreement in anterior and posterior lens curvature, and lens diameter. With deeper signal penetration, the Insight 100 has the advantage of providing a more comprehensive analysis of the lens parameter, especially in the posterior and peripheral lens. In general, the Insight 100 could be a promising tool in measuring lens parameters in vivo, with potential roles in preoperative IOLs selection and postoperative assessment.

\section{DATA AVAILABILITY STATEMENT}

The original contributions presented in the study are included in the article/Supplementary Material, further inquiries can be directed to the corresponding authors.

\section{ETHICS STATEMENT}

The studies involving human participants were reviewed and approved by the Ethics Committee of Zhongshan Ophthalmic Center. The patients/participants provided their written informed consent to participate in this study. Written informed consent was obtained from the individual(s) for the publication of any potentially identifiable images or data included in this article.

\section{REFERENCES}

1. Dubbelman M, Van der Heijde GL, Weeber HA. Change in shape of the aging human crystalline lens with accommodation. Vis Res. (2005) 45:117-32. doi: 10.1016/j.visres.2004.07.032

2. Martinez-Enriquez E, Pérez-Merino P, Velasco-Ocana M, Marcos S. OCTbased full crystalline lens shape change during accommodation in vivo. Biomed Optics Express. (2017) 8:918-33. doi: 10.1364/BOE.8.000918

3. Modesti M, Pasqualitto G, Appolloni R, Pecorella I, Sourdille P. Preoperative and postoperative size and movements of the lens capsular bag: ultrasound biomicroscopy analysis. J Cataract Refract Surg. (2011) 37:1775-84. doi: 10.1016/j.jcrs.2011.04.035

4. Li S, Li X, He S, Zheng Q, Chen X, Wu X, et al. Early Postoperative Rotational stability and its related factors of a single-piece acrylic toric intraocular lens. Eye. (2020) 34:474-9. doi: 10.1038/s41433-019-0521-0

5. Ruan X, Liu Z, Luo L, Liu Y. The structure of the lens and its associations with the visual quality. BMJ Open Ophthalmol. (2020) 5:e000459. doi: 10.1136/bmjophth-2020-000459

6. Liu Z, Ruan X, Wang W, Liu J, Meng Y, Gu X, et al. Comparison of radius of anterior lens surface curvature measurements in vivo using the anterior segment optical coherence tomography and Scheimpflug imaging. Ann Transl Med. (2020) 8:177. doi: 10.21037/atm.2020.01.100

7. Shoji T, Kato N, Ishikawa S, Ibuki H, Yamada N, Kimura I, et al. In vivo crystalline lens measurements with novel swept-source optical coherent tomography: an investigation on variability of measurement. BMJ Open Ophthalmol. (2017) 1:e000058. doi: 10.1136/bmjophth-2016-00 0058

8. Fukuda S, Ueno Y, Fujita A, Mori H, Tasaki K, Murakami T, et al. Comparison of anterior segment and lens biometric measurements in patients with cataract. Graefes Arch Clin Exp Ophthalmol. (2020) 258:137-46. doi: 10.1007/s00417-019-04482-0

\section{AUTHOR CONTRIBUTIONS}

ZL and LL: study concept and design and study supervision. $\mathrm{XR}, \mathrm{CL}$, and ZL: drafting of the manuscript. LL, ZL, and ZX: critical revision of the manuscript. XT and GJ: statistical analysis. All authors have full access to all the data in the study and take responsibility for the integrity of the data and the accuracy of the data analysis, acquisition, analysis, or interpretation of data, completed and submitted the ICMJE form for disclosure of potential conflicts of interest. All authors contributed to the article and approved the submitted version.

\section{FUNDING}

This study was supported by the National Natural Science Foundation of China (81873675 and 81770905) and the Construction Project of High-Level Hospitals in Guangdong Province (303020102).

\section{SUPPLEMENTARY MATERIAL}

The Supplementary Material for this article can be found online at: https://www.frontiersin.org/articles/10.3389/fmed. 2022.777645/full\#supplementary-material

9. Ramasubramanian V, Glasser A. Objective measurement of accommodative biometric changes using ultrasound biomicroscopy. J Cataract Refract Surg. (2015) 41:511-26. doi: 10.1016/j.jcrs.2014.08.033

10. Li X, Chang P, Li Z, Qian S, Zhu Z, Wang Q, et al. Agreement between anterior segment parameters obtained by a new ultrasound biomicroscopy and a swept-source fourier-domain anterior segment optical coherence tomography. Expert Rev Med Devices. (2020) 17:1333-40. doi: 10.1080/17434440.2020.1848541

11. Koeppl C, Findl O, Kriechbaum K, Sacu S, Drexler W. Change in IOL position and capsular bag size with an angulated intraocular lens early after cataract surgery. J Cataract Refract Surg. (2005) 31:348-53. doi: $10.1016 /$ j.jcrs.2004.04.063

Conflict of Interest: The authors declare that the research was conducted in the absence of any commercial or financial relationships that could be construed as a potential conflict of interest.

Publisher's Note: All claims expressed in this article are solely those of the authors and do not necessarily represent those of their affiliated organizations, or those of the publisher, the editors and the reviewers. Any product that may be evaluated in this article, or claim that may be made by its manufacturer, is not guaranteed or endorsed by the publisher.

Copyright (C) 2022 Ruan, Liang, Xia, Tan, Jin, Jin, Liu and Luo. This is an open-access article distributed under the terms of the Creative Commons Attribution License (CC $B Y)$. The use, distribution or reproduction in other forums is permitted, provided the original author(s) and the copyright owner(s) are credited and that the original publication in this journal is cited, in accordance with accepted academic practice. No use, distribution or reproduction is permitted which does not comply with these terms. 